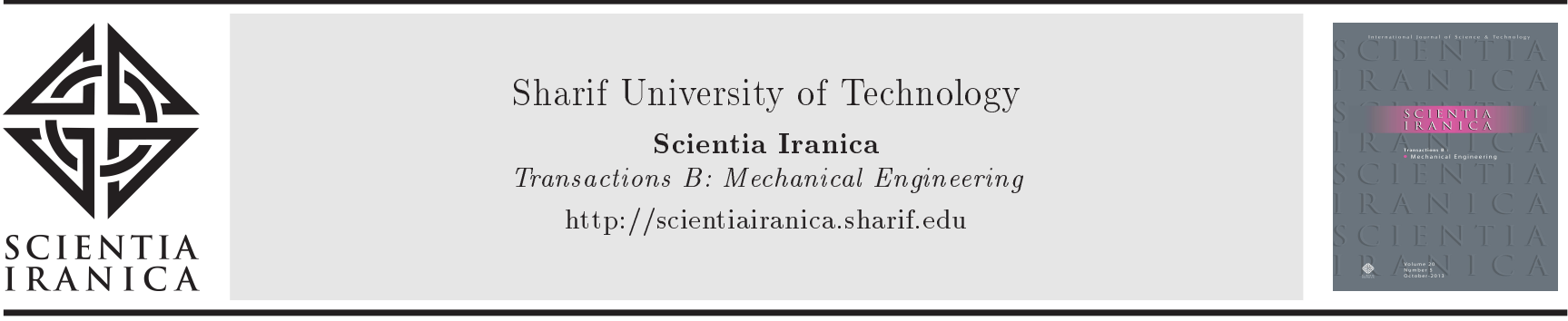

Research Note

\title{
Model reference adaptive control of a small satellite in the presence of parameter uncertainties
}

\author{
K. Ahmadi Dastgerdi ${ }^{\mathrm{a}}$, F. Pazooki ${ }^{\mathrm{a}, *}$, and J. Roshanian ${ }^{\mathrm{b}}$ \\ a. Department of Mechanical and Aerospace Engineering, Science and Research Branch, Islamic Azad University, Tehran, Iran. \\ b. Department of Aerospace Engineering, K.N. Toosi University of Technology, Tehran, Iran.
}

Received 14 February 2018; received in revised form 5 February 2019; accepted 10 August 2019

\author{
KEYWORDS \\ Discrete-time; \\ Model Reference \\ Adaptive Control \\ (MRAC); \\ Parameter \\ uncertainty; \\ Satellite.
}

\begin{abstract}
An accurate control algorithm for small satellites is critical to mission success. In this paper, a novel discrete-time Model Reference Adaptive Control (MRAC) algorithm is developed based on a unified approach for the attitude control of a three-axis stabilized nonlinear satellite model. The linearized model of a satellite with unknown dynamic parameters is derived and a Recursive Least Squares (RLS) algorithm is used to identify the linear model's unknown parameters. In order to take into account the nonlinear model of satellite dynamics, the proposed MRAC strategy is used considering the linear model, the estimation error; and the difference between the actual nonlinear system and the linear model outputs. The actual nonlinear model of the satellite includes moments of inertia uncertainties, external disturbances, and sensor noise on the outputs. The introduced controller performance is compared with a conventional discrete -time MRAC which demonstrates excellent simultaneous regulation and tracking capabilities.
\end{abstract}

(C) 2020 Sharif University of Technology. All rights reserved.

\section{Introduction}

Designing a proper algorithm to control a satellite for accomplishing various missions constitutes a large portion of design activity. As a result, various control strategies ranging from classical to advanced methods have been developed and applied to satellites $[1,2]$. Due to unknown environmental conditions and parameter uncertainties in the dynamics of the satellite model, classical controllers are not so appropriate. To overcome such problems, robust controllers, such as $H_{\infty}$,

\footnotetext{
*. Corresponding author.

E-mail addresses: karimahmadidastgerdi@gmail.com (K. Ahmadi Dastgerdi); pazooki_fa@srbiau.ac.ir (F. Pazooki); roshanian@kntu.ac.ir (J.Roshanian)
}

have been proposed to alleviate the effect of parameter uncertainty [3-6]. However, robust controllers, in general, do not exhibit appropriate performance when considering large parameter variations. In this case, to improve satellite response while encountering large parameter changes, adaptive control methods have been proposed [7]. The instabilities of adaptive controllers have been demonstrated under several conditions [810]. The use of a projection function in adaptive control is a common method which guarantees bounded parameter estimation in control of a satellite $[11,12]$. Another method to make an adaptive controller robust, is the dead zone method [13].

To improve the performance of adaptive control, combined algorithms, such as fuzzy-adaptive, sliding mode-adaptive or neural network adaptive algorithms, have been proposed [14-17], although, implementing these methods is not simple. In Ref. [18], an adaptive 
sliding mode trajectory tracking controller for a cooperative spacecraft rendezvous with coupled uncertain dynamics is designed.

To enhance the performance of the satellite in the presence of reaction wheel faults, an adaptive control algorithm has been proposed in Ref. [19].

Obviously, implementation of adaptive controllers on electrical boards necessitates the adaptive law to be designed in discrete-time. Backstepping is a common discrete-time adaptive method which has been used for satellite attitude control [20-22]. Cruz and Bernstein designed and implemented various model reference adaptive controllers in a discrete-time model [23].

Landau introduced a control strategy called the "unified approach" which is inherently based on linear models [24]. A unified approach controller based on the nonlinear model of a satellite is designed in Ref. [25] by applying linear regression to identify the parameters of the nonlinear model. Despite the above described efforts, the lack of robustness of the identification algorithm is proved in Ref. [13]. Extensive computational calculations have been the biggest obstacle in the development of adaptive controllers in the past. Processor development is one of the reasons that the designers were willing to apply adaptive controllers.

In this paper, a discrete-time Model Reference Adaptive Control algorithm (MRAC) is designed based on a unified approach for a three-axis stabilized nonlinear satellite model in the presence of uncertainty in the inertia matrix, external disturbance, and sensor noise.

The outline of this paper is organized as follows: In Section 2, the satellite's dynamic equations are introduced. The nonlinear MRAC law based on the unified approach is introduced in Section 3, and in Section 4, the simulation results are presented. Finally, the key conclusions are presented in Section 5 .

\section{Satellite equations of motion}

The angular momentum equation of the satellite rigid body about its center of mass is simply given as Eq. (1):

$$
\overrightarrow{\mathbf{T}}=\dot{\overrightarrow{\mathbf{H}}},
$$

where $\overrightarrow{\mathbf{H}}=\mathbf{J} \overrightarrow{\boldsymbol{\omega}}^{\mathbf{B} / \mathbf{E}}$ is the angular momentum vector of the satellite rigid body about its mass center and $\overrightarrow{\mathbf{T}}$ is the external moment acting on the satellite body. $\overrightarrow{\boldsymbol{\omega}}^{\mathrm{B} / \mathbf{E}}$ is the angular velocity vector of the body frame with respect to the Inertial reference frame, and:

$$
\mathbf{J}=\left[\begin{array}{lll}
I_{x x} & I_{x y} & I_{x z} \\
I_{x y} & I_{y y} & I_{y z} \\
I_{x z} & I_{y z} & I_{z z}
\end{array}\right]
$$

is the moments of inertia of the satellite's body. $\dot{\overrightarrow{\mathbf{H}}}$ is defined as below [26]:

$$
\dot{\overrightarrow{\mathbf{H}}} \equiv\left\{\frac{d}{d t} \overrightarrow{\mathbf{H}}\right\}_{E}=\left\{\frac{d}{d t} \overrightarrow{\mathbf{H}}\right\}_{B}+\overrightarrow{\boldsymbol{\omega}}^{\mathbf{B} / \mathbf{E}} \times \overrightarrow{\mathbf{H}} .
$$

By defining $\overrightarrow{\boldsymbol{\omega}}^{\mathrm{B} / \mathbf{E}}=\overrightarrow{\boldsymbol{\omega}}$ and applying Eq. (2), the aforementioned Eq. (1) becomes [26]:

$$
\begin{aligned}
\overrightarrow{\mathbf{T}}= & \left\{\frac{d}{d t} \overrightarrow{\boldsymbol{H}}\right\}_{B}+\overrightarrow{\boldsymbol{\omega}}^{\mathbf{B} / \mathbf{E}} \times \overrightarrow{\mathbf{H}}=\left\{\frac{d}{d t} \mathbf{J}\right\}_{B} \overrightarrow{\boldsymbol{\omega}} \\
& +\mathbf{J}\left\{\frac{d}{d t} \overrightarrow{\boldsymbol{\omega}}\right\}_{B}+\overrightarrow{\boldsymbol{\omega}} \times \mathbf{J} \overrightarrow{\boldsymbol{\omega}},
\end{aligned}
$$

where $\left\{\frac{d}{d t} \mathbf{J}\right\}_{B}=0$ and $\left\{\frac{d}{d t} \overrightarrow{\boldsymbol{\omega}}\right\}_{B}=\left\{\frac{d}{d t} \overrightarrow{\boldsymbol{\omega}}\right\}_{E}=\dot{\overrightarrow{\boldsymbol{\omega}}}$. Therefore, Eq. (4) is obtained [26]:

$$
\overrightarrow{\mathbf{T}}=\mathbf{J} \dot{\vec{\omega}}+\vec{\omega} \times \mathbf{J} \vec{\omega}
$$

The satellite kinematic equation can be presented as below [27]:

$$
\begin{aligned}
{\left[\begin{array}{l}
\omega_{x} \\
\omega_{y} \\
\omega_{z}
\end{array}\right]=} & {\left[\begin{array}{ccc}
S \theta S \psi & C \psi & 0 \\
S \theta C \psi & -S \psi & 0 \\
C \theta & 0 & 1
\end{array}\right]\left[\begin{array}{c}
\dot{\phi} \\
\dot{\theta} \\
\dot{\psi}
\end{array}\right] } \\
& +\left[\begin{array}{ccc}
S \theta S \psi & C \psi & 0 \\
S \theta C \psi & -S \psi & 0 \\
C \theta & 0 & 1
\end{array}\right]\left[\begin{array}{c}
0 \\
-\omega_{0} \\
0
\end{array}\right],
\end{aligned}
$$

where $\phi, \theta$, and $\psi$ (roll, pitch, and yaw angles, respectively) are the Euler angles; and $\omega_{0}$ is orbit angular rate.

\subsection{Environmental disturbance and sensor noise}

Satellites are prone to environmental disturbances including gravity gradient disturbance, aerodynamic disturbance, solar radiation disturbance, etc. Obviously, the main disturbance on a low earth orbit satellite is the gravity gradient disturbance. In this paper, in order to investigate the disturbance effects, it is assumed that the maximum infinity norm of summation of all torque disturbances except the gravity gradient torque is lower than the gravity gradient torque disturbance.

$$
\begin{aligned}
& \overrightarrow{\mathbf{T}} \mathbf{d}=\overrightarrow{\mathbf{T}} \mathbf{g}+\overrightarrow{\mathbf{T}} \mathbf{a}+\overrightarrow{\mathbf{T}} \mathbf{s}+\cdots, \\
& \overrightarrow{\mathbf{T}} \mathbf{d}_{\mathbf{1}}=\overrightarrow{\mathbf{T}} \mathbf{a}+\overrightarrow{\mathbf{T}} \mathbf{s}+\cdots, \\
& \max \left\|\overrightarrow{\mathbf{T}} \mathbf{d}_{\mathbf{1}}\right\|_{\infty} \leq\|\overrightarrow{\mathbf{T}} \mathbf{g}\|_{\infty},
\end{aligned}
$$

where $\overrightarrow{\mathbf{T}} \mathbf{d}$ is the disturbance torque, $\overrightarrow{\mathbf{T}} \mathbf{g}$ is the gravity gradient disturbance and $\overrightarrow{\mathbf{T}} \mathbf{d}_{1}$ is all other disturbances including aerodynamic and solar radiation. Clearly, the 
assumption considered here is quite rational, since the main disturbance, which affects satellites in low Earth orbit, is the gravity gradient disturbance. The worst case of gravity gradient disturbance is presented below:

$$
\|\overrightarrow{\mathbf{T}} \mathbf{g}\|_{\infty}=\frac{3 \mu}{2 R_{e}^{3}}\left(I_{z z}-I_{y y}\right) \sin (2 \Theta),
$$

where $\mu=3.986 \times 10^{14} \frac{\mathrm{m}^{3}}{\mathrm{~s}^{2}}$ is the Earth's gravitational parameter, $R_{e}=6378 \mathrm{~km}$ is the Earth radius, and $\Theta$ is maximum deviation from the vertical axis.

In this paper, it is assumed that the gravity gradient disturbance is known and other disturbances are unknown. The sensor noise considered in this research is a random noise, whose bound is 0.2 .

\section{Discrete-time MRAC design using unified approach}

In the following, a novel MRAC based on a unified approach is designed for a system in which time delays and system order are to be known but the system parameters, including initial conditions (altitude and attitudes) and moments of inertia are unknown.

Accordingly, first, a discrete-time linear model of the time invariant system is derived, and then the adaptive control algorithm is designed based on this model. The discrete linear time-invariant system is considered as below [24]:

$$
\begin{aligned}
& y_{p}(k)=\frac{z^{-t_{d}} B\left(z^{-1}\right)}{A\left(z^{-1}\right)} u(k), \\
& t_{d}>0, \quad y_{p}(0) \neq 0,
\end{aligned}
$$

where $z^{-1}$ is a backward shift operator. $y_{p}(k), u(k)$, and $t_{d}$ are the system's output, input, and time delay, respectively. $B\left(z^{-1}\right)$ and $A\left(z^{-1}\right)$ are the polynomials of the numerator and denominator of the discrete-time transfer function which are unknown in this paper. the polynomials $B\left(z^{-1}\right)$ and $A\left(z^{-1}\right)$ are modeled as below [24].

$$
\begin{aligned}
& B\left(z^{-1}\right)=b_{0}+b_{1} z^{-1}+\cdots+b_{N_{B}} z^{-N_{B}}, \quad b_{0} \neq 0 \\
& A\left(z^{-1}\right)=1+a_{1} z^{-1}+a_{2} z^{-2}+\cdots+a_{N_{A}} z^{-N_{A}}
\end{aligned}
$$

In our novel MRAC, the reference model is defined as:

$$
y_{m}(k)=z^{-t_{d}} \frac{F\left(z^{-1}\right)}{C_{1}\left(z^{-1}\right)} r(k),
$$

where $C_{1}\left(z^{-1}\right)$ and $F\left(z^{-1}\right)$ are polynomials of the numerator and denominator of the reference model transfer function. $C_{1}\left(z^{-1}\right)$ is asymptotically stable, $r(k)$ is a bounded reference sequence, and $y_{m}(k)$ is the output of the reference model. In order to track the desired model reference, the system output $\left(y_{p}(k)\right)$ must satisfy Eq. (11), therefore [24]:

$$
C_{1}\left(z^{-1}\right) y_{p}(k)=z^{-t_{d}} F\left(z^{-1}\right) r(k) .
$$

Hence, the error equation is defined as:

$$
e(k)=y_{p}(k)-y_{m}(k) \text {. }
$$

Obviously, for the tracking objective where $y_{p}(k)$ must track $y_{m}(k)$, the following equation holds [24]:

$$
C_{2}\left(z^{-1}\right) e(k+t d)=0, \quad k>0,
$$

where $C_{2}\left(z^{-1}\right)$ is an asymptotic stable polynomial as described in Eq. (15) [24].

$$
C_{2}\left(z^{-1}\right)=1+\gamma_{1} z^{-1}+\gamma_{2} z^{-2}+\cdots+\gamma_{n} z^{-N c_{2}} .
$$

For the objective of regulation $\left(y_{m}(k)\right.$ is a constant), the control system must reject any disturbance in the initial moment $\left(y_{p}(0) \neq 0\right)$. Therefore, the equation below must be satisfied [28]:

$$
C_{2}\left(z^{-1}\right) y_{p}(k+t d)=0, \quad k \geq 0 .
$$

For a plant with unknown parameters, the recursive least squares error algorithm is a common identification algorithm, which has been implemented in this paper. Therefore, The estimated output will be computed as follows [28]:

$$
\hat{y}(k)=\Phi(k) \hat{\Theta}(k),
$$

where "^" represents the estimation parameters. In Eq. (17), $\hat{y}(k), \hat{\Theta}(k)$, and $\Phi(k)$ represent the estimated outputs, estimated parameters, and regression vectors, respectively, defined according to Eqs. (18) and (19):

$$
\begin{aligned}
& \hat{\Theta}(k)= {\left[\hat{a}_{1}(k) \hat{a}_{2}(k), \cdots, \hat{a}_{N_{A}}(k),\right.} \\
&\left.\hat{b}_{0}(k), \hat{b}_{1}(k), \cdots, \hat{b}_{N_{B}}(k)\right]^{T}, \\
& \Phi(k)=\left[-y_{p}(k-1), \cdots,-y_{p}\left(k-N_{A}\right),\right. \\
&\left.u(k-1), \cdots, u\left(k-N_{B}\right)\right] .
\end{aligned}
$$

The estimation error is defined as:

$$
\varepsilon(k)=y_{L p}(k)-\hat{y}(k),
$$

where $y_{L p}(k)$ is the output of the satellite linear model (Eq. (8)). According to Eq. (20) the linear model's output has been obtained as below:

$$
y_{L p}(k)=\hat{y}(k)+\varepsilon(k) \text {. }
$$

By substituting Eq. (21) in to Eq. (14) and using Eqs. (18) and (19), the control law is obtained as follow: 


$$
\begin{aligned}
u(k)= & \frac{1}{\hat{b}_{0}}\left(C_{2}\left(z^{-1}\right) y_{m}(k+t d)-\mu_{1}\left(z^{-1}\right) \hat{y}(k)\right. \\
& \left.-\mu_{2}\left(z^{-1}\right) u(k)+C_{2}\left(z^{-1}\right) \varepsilon(k)\right),
\end{aligned}
$$

where:

$$
\begin{aligned}
& C_{2}\left(z^{-1}\right)=A\left(z^{-1}\right) S\left(z^{-1}\right)+z^{-t_{d}} \mu_{1}\left(z^{-1}\right), \\
& N c_{2}=N_{A}+t_{d}-1, \\
& S\left(z^{-1}\right)=1+s_{1} z^{-1}+\cdots+s_{n_{s}} z^{-N_{s}}, \quad N_{S}=t_{d}-1, \\
& \mu_{1}\left(z^{-1}\right)=\lambda_{0}+\lambda_{1} z^{-1}+\cdots+\lambda_{N_{\mu_{1}}} z^{-N_{\mu_{1}}}, \\
& N_{\mu_{1}}=\max \left(N_{A}-1, N c_{2}-t_{d}\right), \\
& \mu_{2}\left(z^{-1}\right)=B\left(z^{-1}\right) S\left(z^{-1}\right)-b_{0} .
\end{aligned}
$$

The block diagram architecture of the controller is shown in Figure 1.

The first novel modification of the control algorithm which has not been previously implemented in the unified approach is the augmentation of error estimation in the control law.

\subsection{Problem definition}

In this section, a MRAC is designed for a linear three-axis stabilized satellite. Then, it is extended for the nonlinear dynamic equation of motion in the presence of the axes coupling, un-modeled dynamics, external disturbances, and sensor noise. Accordingly, the satellite attitude dynamic equations are initially linearized as Eq. (24) [27]:

$$
\left\{\begin{array}{l}
\ddot{\phi}=\frac{T_{x}-4 \omega_{0}^{2}\left(I_{y y}-I_{z z}\right) \phi}{I_{x x}} \\
\ddot{\theta}=\frac{T_{y}-3 \omega_{0}^{2}\left(I_{x x}-I_{z z}\right) \theta}{I_{y y}} \\
\ddot{\psi}=\frac{T_{z}-\omega_{0}^{2}\left(I_{y y}-I_{x x}\right) \psi}{I_{z z}}
\end{array}\right.
$$

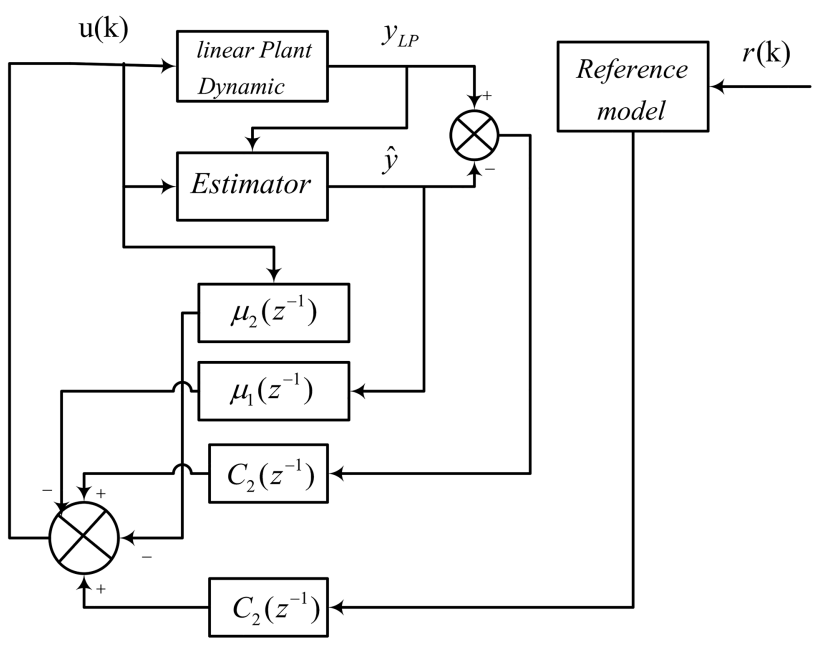

Figure 1. Control algorithm block diagram.
Obviously, there is a difference between Eq. (24) and satellite's actual output.

$$
\overrightarrow{\mathbf{y}}_{\mathbf{N}}-\overrightarrow{\mathbf{y}}_{\mathbf{L P}}=\vec{\Delta}
$$

where $\overrightarrow{\mathbf{y}}_{\mathbf{N}}$ is the output vector obtained from the satellite's actual output and $\overrightarrow{\mathbf{y}}_{\mathbf{L P}}$ is the output vector derived from the linear equation of motion equation (24). Since a linear model is used in the adaptive controller design, it is necessary to apply the vector $\overrightarrow{\boldsymbol{\Delta}}=\left[\begin{array}{lll}d_{1} & d_{2} & d_{3}\end{array}\right]^{T}$ in the control algorithm. In other words, the nonlinear equation of motion is rewritten as Eq. (26):

$$
\begin{aligned}
& y_{p i}(k)=\frac{z^{-t_{d}} B_{i}\left(q^{-1}\right)}{A_{i}\left(q^{-1}\right)} u_{i}(k)+d_{i}, \\
& t_{d}>0, \quad y_{p i}(0) \neq 0, \quad i=1,2,3,
\end{aligned}
$$

where:

$$
\begin{gathered}
\left\{\begin{array}{l}
y_{p_{1}}(k)=\phi(k) \\
y_{p_{2}}(k)=\theta(k) \\
y_{p_{3}}(k)=\psi(k)
\end{array}\right. \\
\left\{\begin{array}{l}
u_{1}(k)=T_{x}(k) \\
u_{2}(k)=T_{y}(k) \\
u_{3}(k)=T_{z}(k)
\end{array}\right.
\end{gathered}
$$

The nominator and denominator of Eq. (26) are:

$$
\begin{aligned}
& B_{i}\left(z^{-1}\right)=b_{0 i}+b_{1 i} z^{-1} \\
& A_{i}\left(z^{-1}\right)=1+a_{1 i} z^{-1}+a_{2 i} z^{-2} .
\end{aligned}
$$

According to Eqs. (14), (22), (24) and (26), the input vector is obtained as Eq. (31):

$$
\begin{aligned}
u_{i}(k)= & \frac{1}{\hat{b}_{0 i}}\left[C_{2}\left(z^{-1}\right) y_{m i}\left(k+t_{d}\right)-\mu_{1}\left(z^{-1}\right) \hat{y}_{i}(k)\right. \\
& -\mu_{2}\left(z^{-1}\right) u_{i}(k)+C_{2}\left(z^{-1}\right) \varepsilon_{i}(k) \\
& \left.+C_{2}\left(z^{-1}\right) d_{i}(k)\right]
\end{aligned}
$$

The second modification on the control law which causes considerable performance enhancement, is to add the effect of the $\overrightarrow{\boldsymbol{\Delta}}=\left[\begin{array}{lll}d_{1} & d_{2} & d_{3}\end{array}\right]^{T}$ vector in the control law of Eq. (22). The two-step modification on the unified approach introduced in this paper has not been previously applied in other literatures.

The block diagram of the control system is illustrated in Figure 2.

The proposed control strategy stability can be easily proved using Popov's method [29].

\subsection{Stability proof}

Theorem: The hyper stability concept deals mainly 


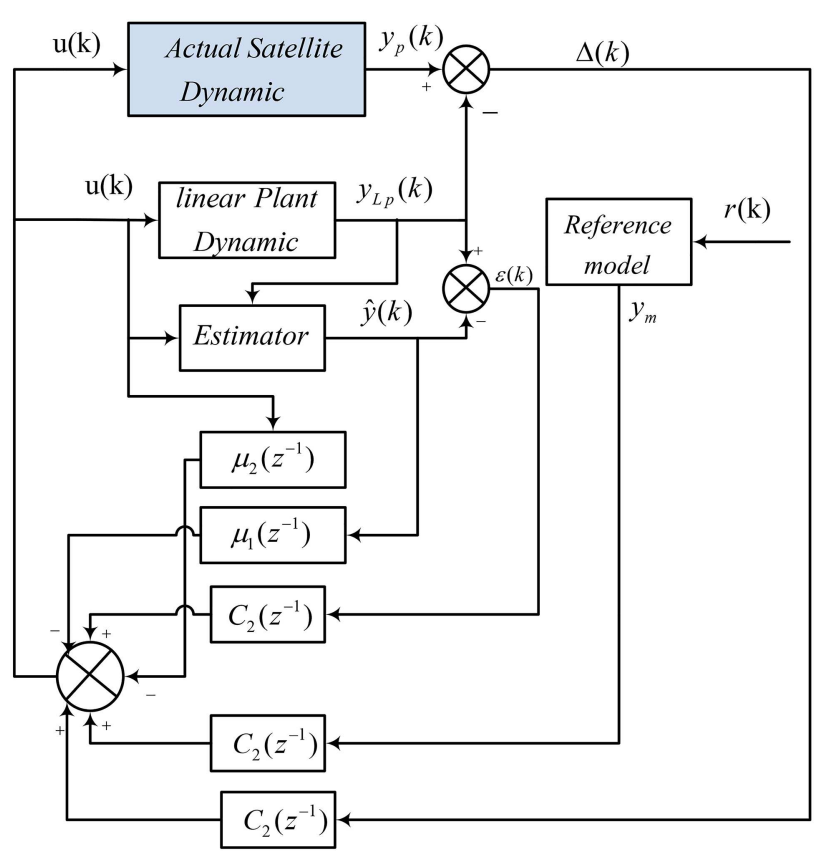

Figure 2. Control algorithm block diagram.

with the stability properties of standard feedback control systems, which satisfies the below inequality:

$$
\sum_{k=k_{0}}^{k_{1}} u^{T}(k) y(k) \geq-\gamma_{0}^{2} \quad \text { for all } \quad k_{1} \geq k_{0} .
$$

Considering Popov's hyperstability, the necessary and sufficient condition for asymptotic stability of a general transfer function of a linear system $G\left(z^{-1}\right)$, with a standard feedback controller is that $G\left(z^{-1}\right)$ must be strictly positive real.

\subsubsection{Stability of the designed algorithm}

According to Eqs. (21), (25), (29), and (30), the output of the satellite equation of motion is:

$$
\begin{aligned}
y_{p i}(k)= & \hat{b}_{0 i} u_{i}(k)+\hat{b}_{1 i} u_{i}(k-1)-\hat{a}_{1 i} z^{-1} y_{p i}(k-1) \\
& -\hat{a}_{2 i} z^{-2} y_{p i}(k-2)+\varepsilon_{i}(k)+d_{i}(k),
\end{aligned}
$$

where:

$$
\begin{array}{r}
y_{p i}(k)=\left\{\begin{array}{lll}
\phi(k) & \text { if } & i=1 \\
\theta(k) & \text { if } & i=2 \\
\psi(k) & \text { if } & i=3
\end{array}\right. \\
u_{i}(k)=\left\{\begin{array}{lll}
T_{x}(k) & \text { if } & i=1 \\
T_{y}(k) & \text { if } & i=2 \\
T_{z}(k) & \text { if } & i=3
\end{array}\right.
\end{array}
$$

By using Eqs. (13), (31), (33), and (34) the error is obtained as below:

$$
\begin{aligned}
& \left\{\begin{array}{l}
e_{1}(k)=\rho_{11} e_{1}(k-1)+\rho_{12} e_{1}(k-2)+\Gamma_{1}(k) \\
e_{2}(k)=\rho_{21} e_{2}(k-1)+\rho_{22} e_{2}(k-2)+\Gamma_{2}(k) \\
e_{3}(k)=\rho_{31} e_{3}(k-1)+\rho_{32} e_{3}(k-2)+\Gamma_{3}(k)
\end{array}\right. \\
& \left\{\begin{array}{l}
G_{1}\left(z^{-1}\right)=\frac{e_{1}(k)}{\Gamma_{1}(k)}=\frac{1}{1-\rho_{11} z^{-1}-\rho_{12} z^{-2}} \\
G_{2}\left(z^{-1}\right)=\frac{e_{2}(k)}{\Gamma_{2}(k)}=\frac{1}{1-\rho_{21} z^{-1}-\rho_{22} z^{-2}} \\
G_{3}\left(z^{-1}\right)=\frac{e_{3}(k)}{\Gamma_{3}(k)}=\frac{1}{1-\rho_{31} z^{-1}-\rho_{32} z^{-2}}
\end{array}\right.
\end{aligned}
$$

As a result of Popov's hyperstability theory, for asymptotic stability $G_{1}\left(z^{-1}\right), G_{2}\left(z^{-1}\right)$, and $G_{3}\left(z^{-1}\right)$ must be strictly positive real. Therefore, the following conditions must be satisfied:

1. The error transfer functions (Eq. (36)) must be positive real;

2. $\Gamma_{i}(k), \Gamma_{2}(k)$, and $\Gamma_{3}(k)$ must be bounded.

The stability of the estimation algorithm has been proved in [29]. The first condition of stability is achieved by appropriate selections of the model reference and $C_{2}\left(z^{-1}\right)$. Since the external disturbances, sensor noise, and the un-modeled dynamic are bounded, $\Gamma_{1}(k), \Gamma_{2}(k)$ and $\Gamma_{3}(k)$ are consequently bounded.

According to the satellite dynamics and achieving the stability purpose of the control law, the $C_{1}\left(z^{-1}\right)$ and $C_{2}\left(z^{-1}\right)$ are considered as order 2. Therefore, the desired poles of $C_{1}\left(z^{-1}\right)$ and $C_{2}\left(z^{-1}\right)$ polynomials are selected as $\{0.07,0.05\}$ and $\{0.01,0.01\}$ respectively, Thus:

$$
\begin{aligned}
& C_{1}\left(z^{-1}\right)=1-0.12 z^{-1}+0.0035 z^{-2} \\
& C_{2}\left(z^{-1}\right)=1-0.02 z^{-1}+10^{-4} z^{-2}
\end{aligned}
$$

To show the effectiveness of the proposed method, a model reference adaptive controller, without considering estimation error and the " $\Delta$ " parameter, is designed here. According to Ref. [24], the control law is defined as below [24]:

$$
\begin{aligned}
u_{i}(k)= & \frac{1}{\hat{b}_{0 i}}\left[C_{2}\left(z^{-1}\right) y_{m i}\left(k+t_{d}\right)-\mu_{1}\left(z^{-1}\right) \hat{y}_{i}(k)\right. \\
& \left.-\mu_{2}\left(z^{-1}\right) u_{i}(k)\right] .
\end{aligned}
$$

\section{Numerical results}

Numerical results of the closed loop tracking maneuver for small roll, pitch and yaw angles with initial conditions $\phi(0)=10^{\circ}, \omega_{x}(0)=4 \frac{\mathrm{deg}}{\mathrm{sec}}, \theta(0)=10^{\circ}$, $\omega_{y}(0)=-4 \frac{\mathrm{deg}}{\mathrm{sec}}, \psi(0)=10^{\circ}$ and $\omega_{z}(0)=4 \frac{\mathrm{deg}}{\mathrm{sec}}$ are illustrated in Figures 3 and 4, which demonstrate the excellent performance of the proposed algorithm. The case study in this paper is a satellite whose moment of inertia is $J=\operatorname{diag}\left[\begin{array}{lll}2.13 & 3.04 & 3.09\end{array}\right] \mathrm{kgm}^{2}$. The 

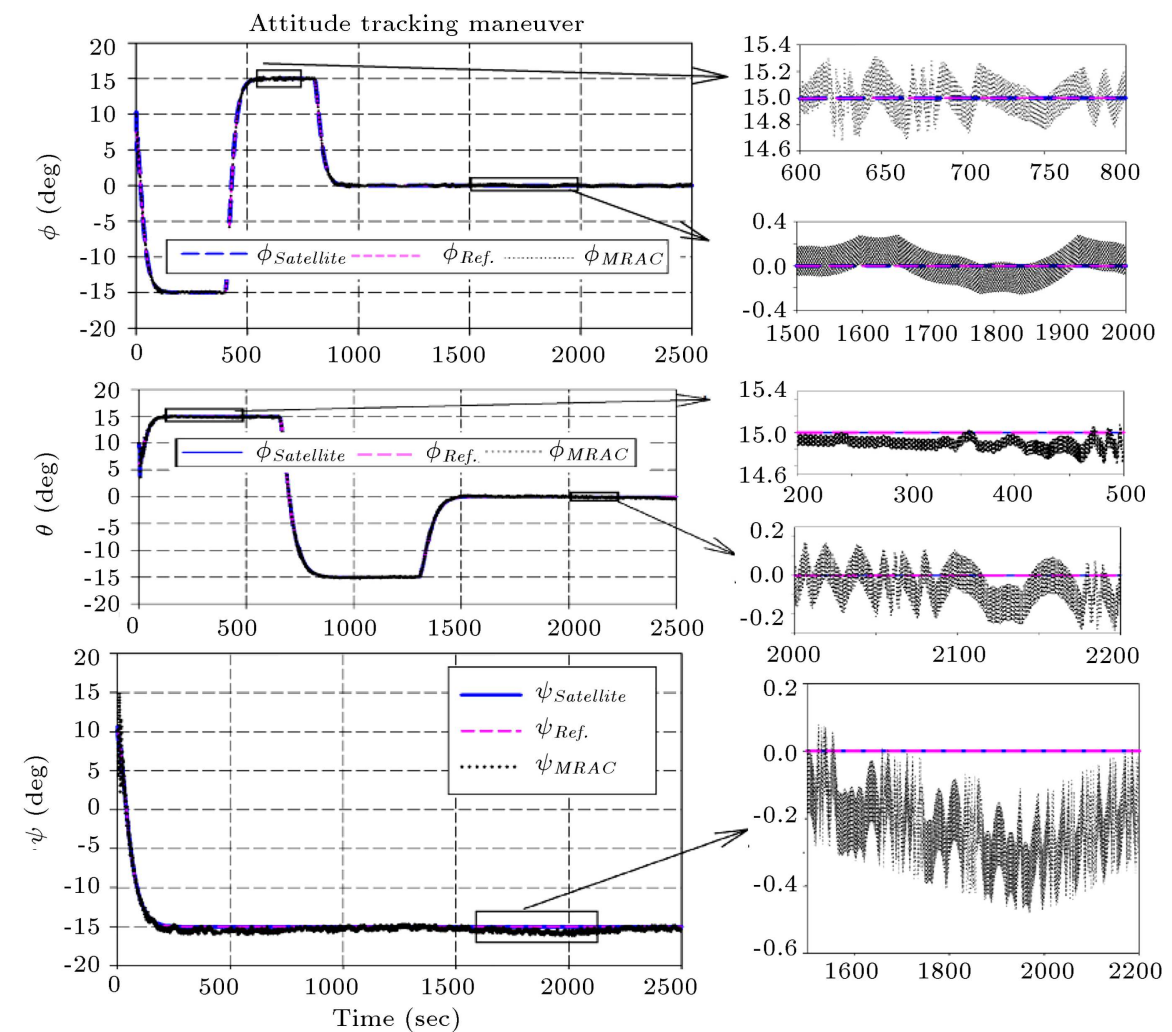

Figure 3. Small angles tracking (comparison of the proposed algorithm with conventional Model Reference Adaptive Control (MRAC) algorithm).
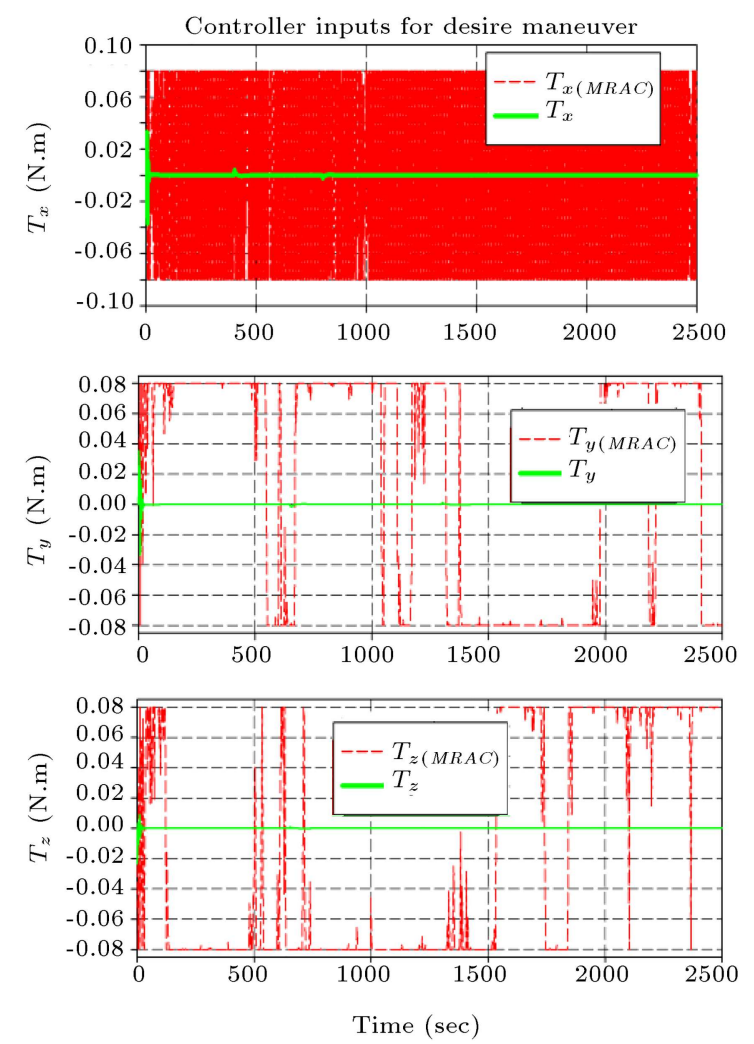

Figure 4. Input torques (comparison of the proposed algorithm with conventional Model Reference Adaptive Control (MRAC) algorithm for small angles tracking). maneuvers presented here are just scenarios which show the designed controller performance and are not necessarily practical scenarios.

In Figure 5, the results for large angle tracking is shown. Figures 3 and 4 illustrate small angle tracking by the conventional MRAC introduced in Ref. [19], (Eq. (38)). As shown, the performance is not suitable i.e., small oscillations in the pitch, roll and yaw are observed and the consumed energy of the controller is too high, i.e. the control effort $\left(\int u^{2} d t\right)$ is too much. Figure 5 shows that tracking larger angles via a conventional MRAC algorithm is not satisfactory, but the proposed algorithm introduced in this article illustrates a desired tracking performance. Therefore, in the following scenarios, only the responses of the proposed algorithm are investigated under several conditions.

In Figure 6 the controlled satellite outputs in the presence of un-molded sensor noise has been illustrated. In this research, the sensor noise bound is assumed to be 0.2 degrees.

Numerical results for $30 \%$ uncertainties in the moments of inertia matrix are shown in Figure 7 .

The results presented in Figure 8 show the proper performance of the MRAC controller in the presence of $30 \%$ uncertainties. $30 \%$ uncertainties in the moment of inertia matrices lead to the controller input oscillation at the beginning of the attitude correction maneuver.

The maximum infinity norm of gravity gradient 

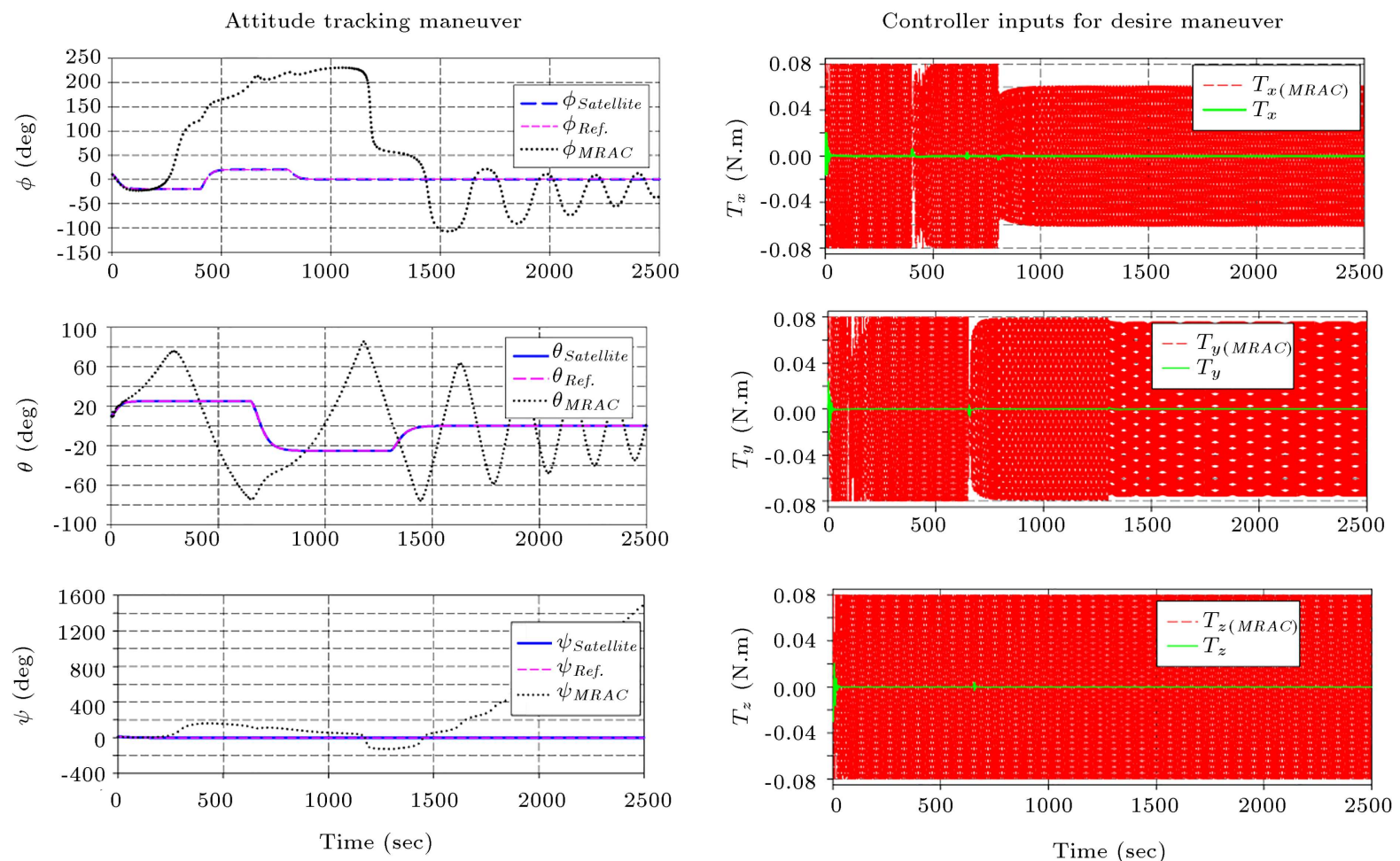

(a)

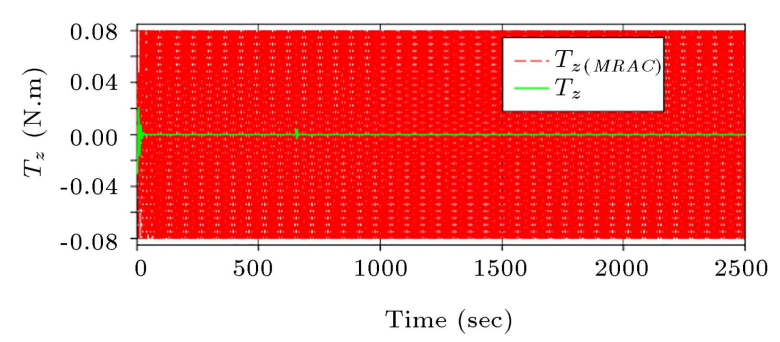

(b)

Figure 5. Comparison of the proposed algorithm with conventional Model Reference Adaptive Control (MRAC) algorithm in large angles tracking: (a) Attitude response and (b) input torques.

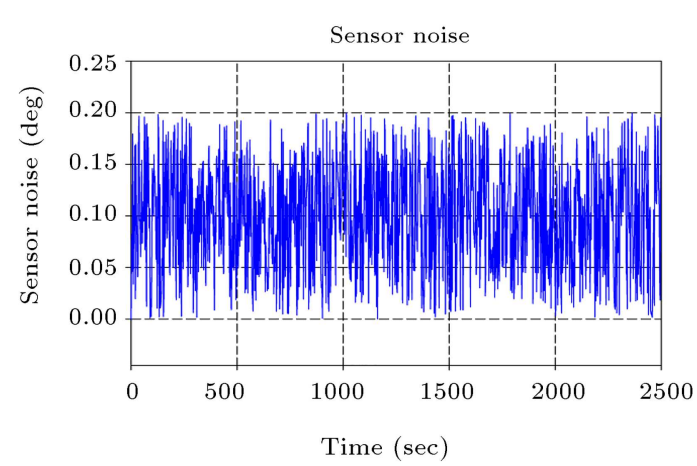

(a)

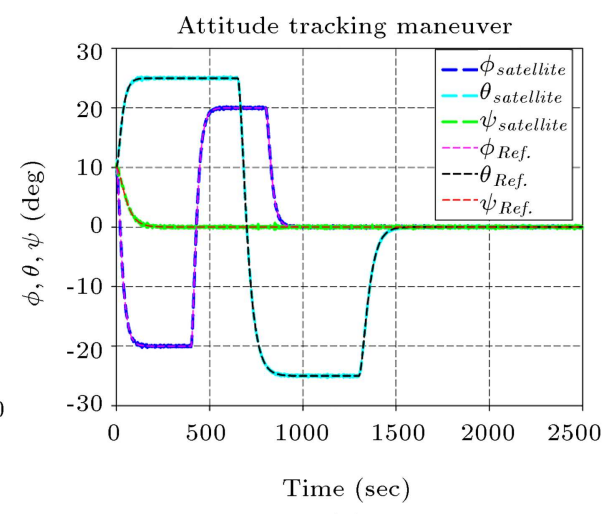

(b)

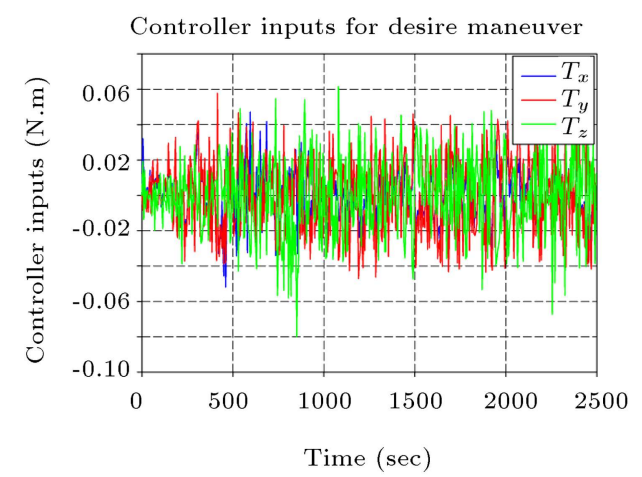

(c)

Figure 6. Satellite performance in the presence of un-molded sensor noise: (a) Un-modeled sensor noise, (b) attitude response, and (c) input torques. 


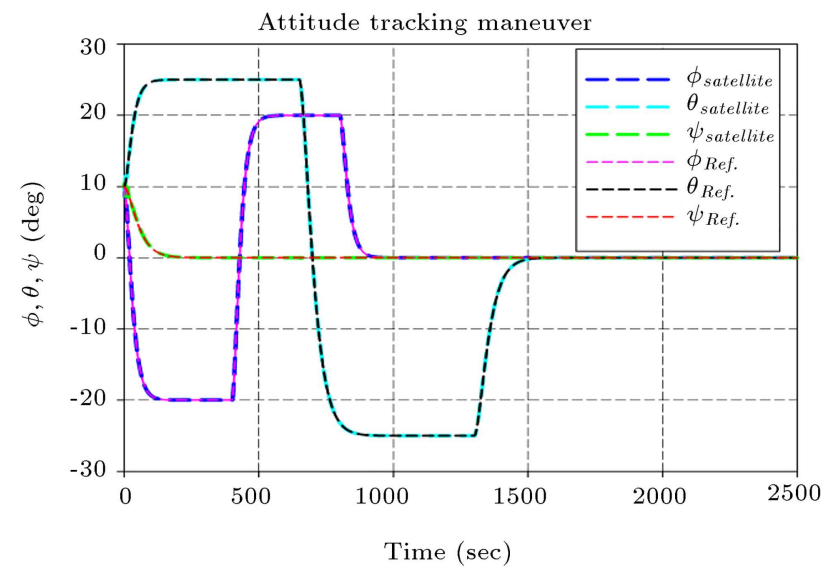

(a)

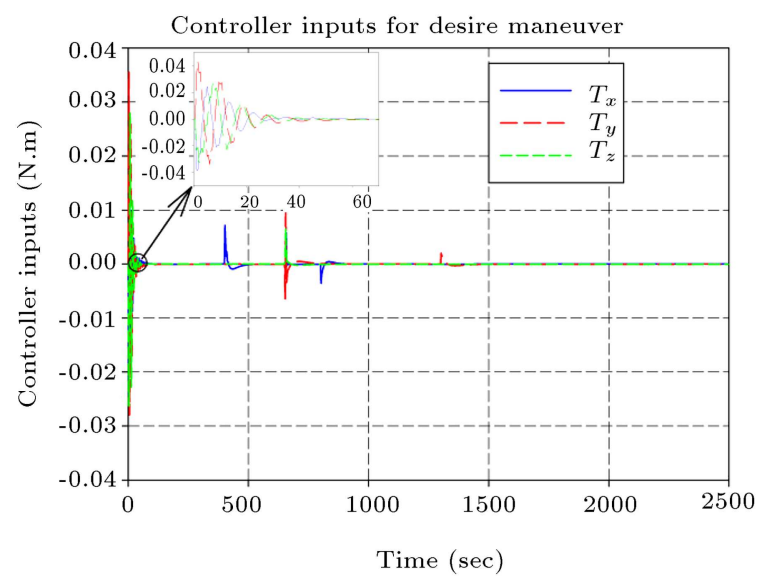

(b)

Figure 7. Satellite performance in the presence of uncertainties: (a) Attitude response and (b) input torques.

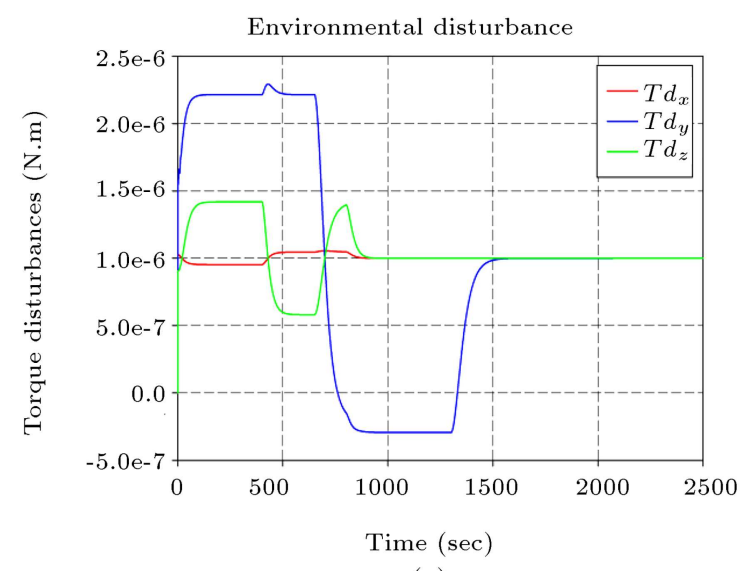

(a)

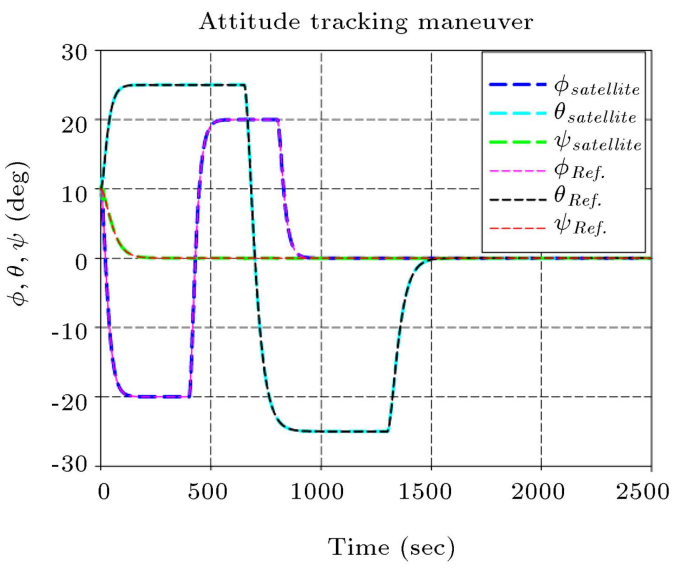

(b)

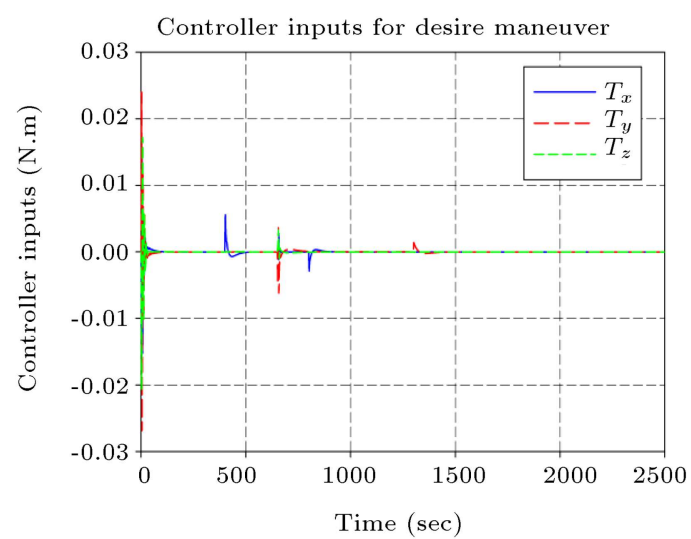

(c)

Figure 8. Satellite performance in the presence of constant and gravity gradient disturbance applied on satellite: (a) Constant and gravity gradient disturbance, (b) attitude response, and (c) input torques.

torque disturbance in the worst case is considered as:

$$
\max (\|\overrightarrow{\mathbf{T}} \mathbf{g}\|)=1 \times 10^{-6} \text { N.m. }
$$

The other disturbance torque, $\overrightarrow{\mathbf{T}} \mathbf{d}_{\mathbf{1}}$, is also assumed to be in different function forms such as constant, sinusoidal, trapezoidal and sawtooth, with the aforementioned maximum value $\left(1 \times 10^{-6}\right.$ N.m $)$. Therefore,
Figures 9-11 illustrate the satellite outputs in the presence of constant, sinusoidal, trapezoidal and sawtooth environmental disturbances with addition gravity gradient disturbance.

According to Figures 3-11, suitable and acceptable satellite controlled outputs, verifies the excellent performance of the designed algorithm. Additionally, 


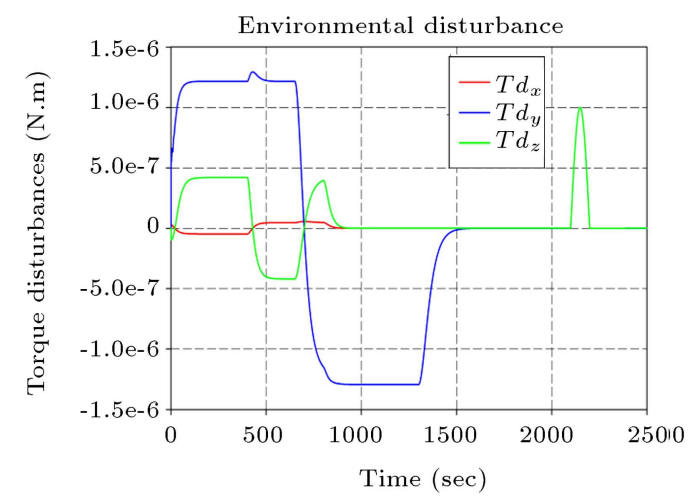

(a)

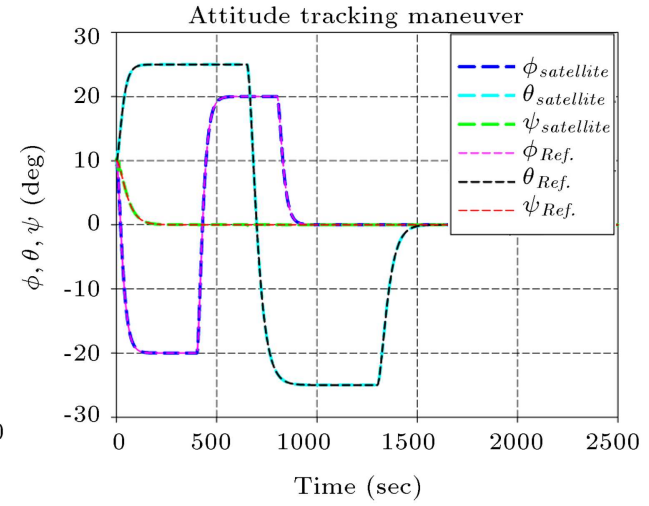

(b)

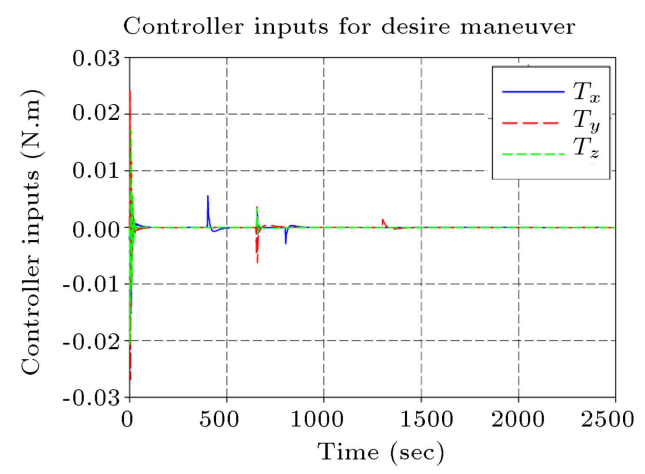

(c)

Figure 9. Satellite performance in the presence of sinusoidal and gravity gradient disturbance applied on satellite: (a) Sinusoidal and gravity gradient disturbance, (b) attitude response, and (c) input torques.

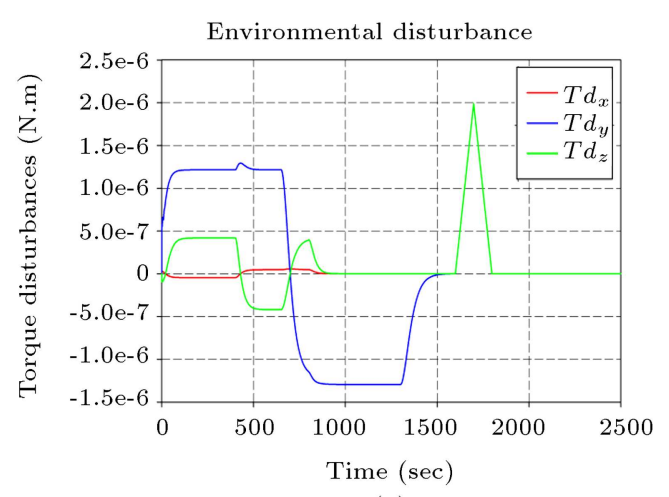

(a)

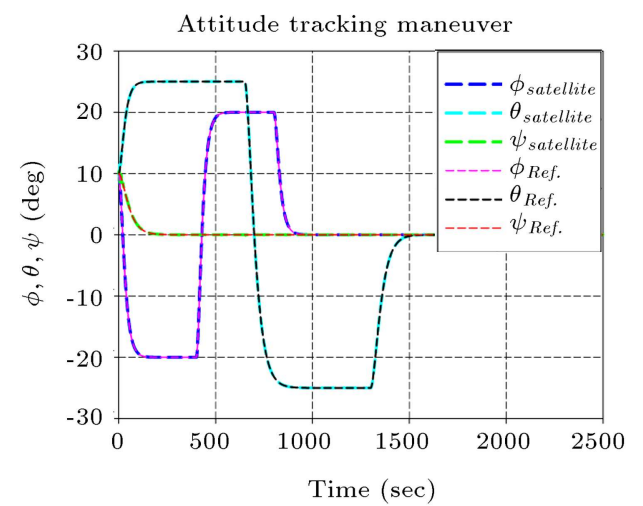

(b)

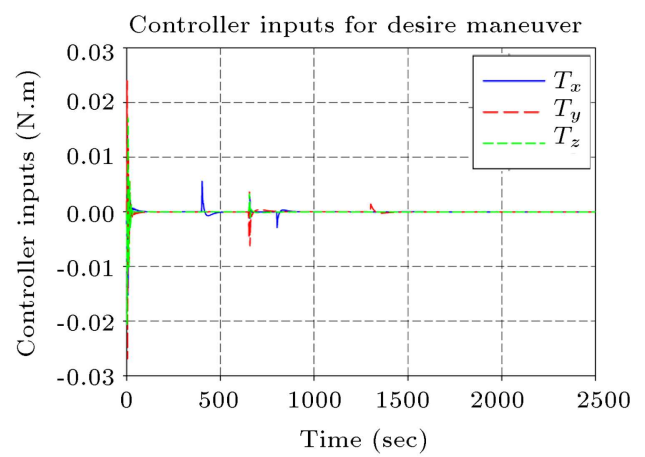

(c)

Figure 10. Satellite performance in the presence of trapezoidal and gravity gradient disturbance applied on satellite: (a) Trapezoidal and gravity gradient disturbance, (b) attitude response, and (c) input torques. 


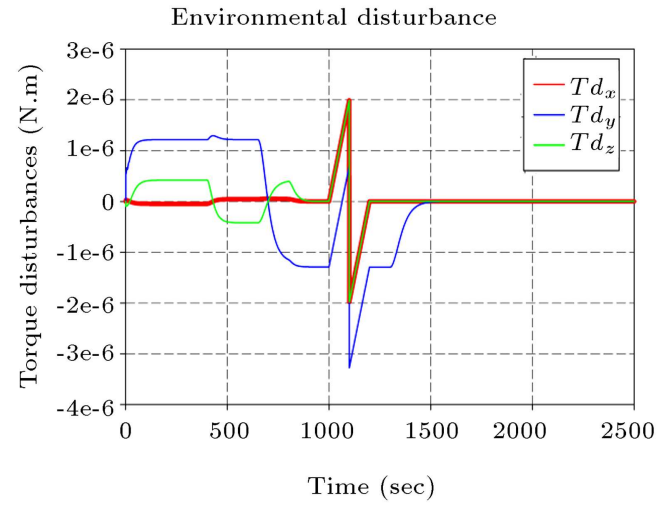

(a)

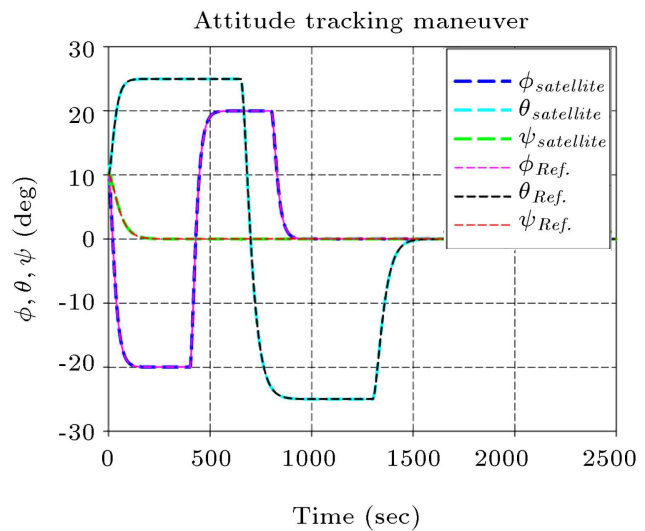

(b)

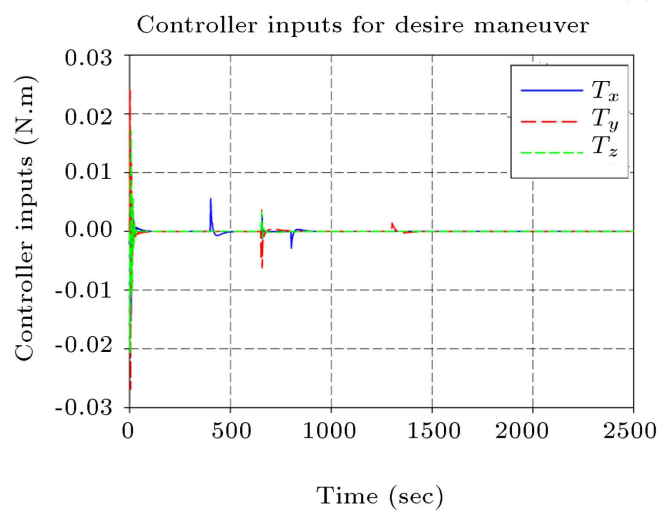

(c)

Figure 11. Satellite performance in the presence of sawtooth and gravity gradient disturbance applied on satellite: (a) Sawtooth and gravity gradient disturbance, (b) attitude response, and (c) input torques.

the designed algorithm is able to successfully eliminate the environmental disturbance effects on the outputs.

\section{Conclusion}

In this paper, a new discrete-time adaptive control for a 3 -axis stabilized satellite is designed. In this algorithm, with the addition of the difference parameters between the linear and nonlinear model, $d_{i}$ in the controller algorithm and addition of the estimation error to the control law, the effect of disturbances and unmolded dynamics have been properly decreased. The designed controller's performance for the prescribed motion is effective, and results from simulation of the control algorithm in the presence of uncertainties, un-modeled sensor noise and environmental disturbance, prove its performance and accuracy; while, conventional Model Reference Adaptive Control (MRAC) exhibits poor performance even in small angles of pitch, roll and yaw maneuvers. The advantages of this control algorithm are its suitability in tracking, simplicity of implementation, and high adaptation speed. Consequently, it can be concluded that the approach provided in this research is effective.

\section{References}

1. Årzén, K.-E. "A simple event-based PID controller", 14 th IFAC World Congress (1999).

2. Psiaki, M.L. "Magnetic torquer attitude control via asymptotic periodic linear quadratic regulation", Journal of Guidance, Control, and Dynamics, 24(2), pp. 386-394 (2001).

3. Binette, M.R., Damaren, C.J., and Pavel, L. "Nonlinear $H_{\infty}$ attitude control using modified Rodrigues parameters", Journal of Guidance, Control, and Dynamics, 37(6), pp. 2017-2021 (2014).

4. Liu, C., Vukovich, G., Shi, K., and Sun, Z. "Robust fault tolerant nonfragile $H_{\infty}$ attitude control for spacecraft via stochastically intermediate observer", Advances in Space Research, 62(9), pp. 2631-2648 (2018).

5. Giri, D.K. and Sinha, M. "Robust backstepping magnetic attitude control of satellite subject to unsymmetrical mass properties", Journal of Spacecraft and Rockets, 56(1), pp. 298-305 (2019).

6. Mori, K. and Takahashi, M. "Minimum-time attitude maneuver and robust attitude control of small satellite mounted with communication antenna", AIA A Scitech 
2019 Forum, American Institute of Aeronautics and Astronautics (2019).

7. Lu, K. and Xia, Y. "Adaptive attitude tracking control for rigid spacecraft with finite-time convergence", Automatica, 49(12), pp. 3591-3599 (2013).

8. Ioannou, P.A. and Kokotovic, P.V. "Instability analysis and improvement of robustness of adaptive control", Automatica, 20(5), pp. 583-594 (1984).

9. Rohrs, C., Valavani, L., Athans, M., and Stein, G. "Robustness of continuous-time adaptive control algorithms in the presence of unmodeled dynamics", IEEE Transactions on Automatic Control, 30(9), pp. 881-889 (1985).

10. Sastry, S. and Bodson, M., Adaptive Control: Stability, Convergence and Robustness, Courier Corporation (2011).

11. Yoon, H. and Tsiotras, P. "Adaptive spacecraft attitude tracking control with actuator uncertainties", The Journal of the Astronautical Sciences, 56(2), pp. 251268 (2008).

12. Kim, D., MacKunis, W., Fitz-Coy, N., and Dixon, W. "Precision Integrated Power and Attitude Control System (IPACS) in the presence of dynamic uncertainty", The Journal of the Astronautical Sciences, 58(1), pp. 99-120 (2011).

13. Ioannou, P.A. and Sun, J., Robust Adaptive Control, Courier Corporation (2012).

14. Jin, X., Zhu, S., Zhu, X., Chen, Q., and Zhang, $\mathrm{X}$. "Single-input adaptive fuzzy sliding mode control of the lower extremity exoskeleton based on humanrobot interaction", Advances in Mechanical Engineering, 9(2), pp. 1-9 (2017).

15. Jiao, X. and Jiang, J. "Design of adaptive switching control for hypersonic aircraft", Advances in Mechanical Engineering, 7(10), pp. 1-10 (2015).

16. Torabi, M., Sharifi, M., and Vossoughi, G. "Robust adaptive sliding mode admittance control of exoskeleton rehabilitation robots", Scientia Iranica, 25(5), pp. 2628-2642 (2018).

17. Sun, R., Wang, J., Zhang, D., and Shao, X. "Neuralnetwork-based sliding-mode adaptive control for spacecraft formation using aerodynamic forces", Journal of Guidance, Control, and Dynamics, 41(3), pp. 757-763 (2017).

18. Sun, L. and Zheng, Z. "Adaptive sliding mode control of cooperative spacecraft rendezvous with coupled uncertain dynamics", Journal of Spacecraft and Rockets, 54(3), pp. 652-661 (2017).

19. Bolandi, H., Haghparast, M., and Abedi, M. "A reliable fault tolerant attitude control system based on an adaptive fault detection and diagnosis algorithm together with a backstepping fault recovery controller", Scientia Iranica, Transaction D, Computer Science \& Engineering, Electrical, 20(6) (2013).

20. Imran, A., Radice, G., and Kim, J. "Backstepping control design with actuator torque bound for spacecraft attitude maneuver", Journal of Guidance, Control, and Dynamics, 33(1), pp. 254-259 (2010).

21. Jiang Y., Hu Q., and Ma, G. "Adaptive backstepping fault-tolerant control for flexible spacecraft with unknown bounded disturbances and actuator failures", ISA Transactions, 49(1), pp. 57-69 (2010).

22. Sun, L. and Huo, W. "6-DOF integrated adaptive backstepping control for spacecraft proximity operations", IEEE Transactions on Aerospace and Electronic Systems, 51(3), pp. 2433-2443 (2015).

23. Cruz, G. and Bernstein D.S. "Retrospective cost adaptive control of spacecraft attitude using magnetic actuators", Proc. AIAA Guid. Nav. Contr. Conf., Boston, MA (2013).

24. Landau, I. and Lozano, R. "Unification of discrete time explicit model reference adaptive control designs", Automatica, 17(4), pp. 593-611 (1981).

25. Saberi, F.F., Dastgerdi, S.A., and Zandieh, M. "Unified model reference adaptive attitude control of a satellite in presence of uncertain parameters: Design and implementation", International Journal of Computer Applications, 121(12), pp. 25-32 (2015).

26. Wie, B., Space Vehicle Dynamics and Control, AIAA (1998).

27. Sidi, M.J., Spacecraft Dynamics and Control: A Practical Engineering Approach, Cambridge University Press (1997).

28. Chalam, V., Adaptive Control Systems: Techniques and Applications, Marcel Dekker, Inc. (1987).

29. Popov, V.-M., Hyperstability of Control Systems, Springer-Verlag Berlin Heidelberg (1973).

\section{Biographies}

Karim Ahmadi Dastgerdi received his $\mathrm{PhD}$ in Aerospace Engineering from the Science \& Research Branch, IAU, Iran, in 2018. His research interests include Adaptive Control Design.

Farshad Pazooki received his $\mathrm{PhD}$ in Aerospace Engineering from the Science \& Research Branch, IAU, Iran, in 2008, where he is currently Assistant Professor of Aerospace Engineering. His research interests include Aircraft Design, Applied Optimal Control, Optimal Trajectory Design and Analysis of Aerospace Vehicles.

Jafar Roshanian received his BS in Mechanical Engineering from K.N. Toosi University of Technology in 1990 and attended the Moscow State Technical 
University (MSTU) in 1993 to continue his education. He spent six years at MSTU as a postgraduate student and received his $\mathrm{MS}$ and $\mathrm{PhD}$ in Flight Dynamics and Control. He joined the Aerospace Engineering Department of K.N. Toosi University in 1999 where he is presently Professor and Head of the Space System Design Institute. He is also an organizing member of the Iranian Aerospace Society and Editorin-Chief of the Space Science and Technology journal. 